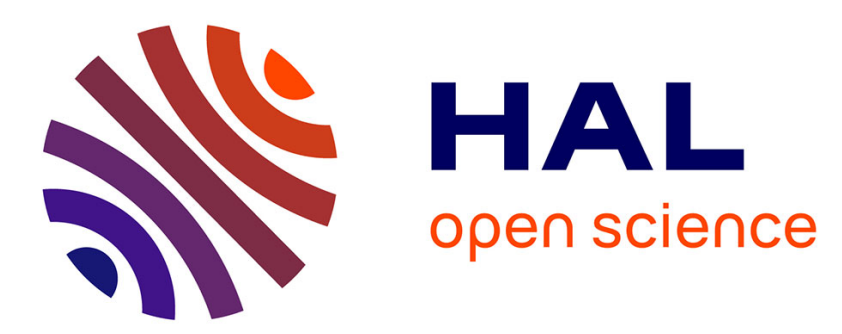

\title{
Validation d'acquis en français sur objectif universitaire (FOU) : quelques pratiques actuelles et propositions à considérer au regard du CECRL
}

Nathalie Gettliffe

\section{- To cite this version:}

Nathalie Gettliffe. Validation d'acquis en français sur objectif universitaire (FOU) : quelques pratiques actuelles et propositions à considérer au regard du CECRL. Recherche et pratiques pédagogiques en langues de spécialité - Cahiers de l'APLIUT, 2013, 32 (1), pp.114-129. 10.4000/apliut.3596 . hal03228234

\section{HAL Id: hal-03228234 \\ https://hal.science/hal-03228234}

Submitted on 17 May 2021

HAL is a multi-disciplinary open access archive for the deposit and dissemination of scientific research documents, whether they are published or not. The documents may come from teaching and research institutions in France or abroad, or from public or private research centers.
L'archive ouverte pluridisciplinaire HAL, est destinée au dépôt et à la diffusion de documents scientifiques de niveau recherche, publiés ou non, émanant des établissements d'enseignement et de recherche français ou étrangers, des laboratoires publics ou privés. 


\section{Validation d'acquis en français sur objectif universitaire (FOU) : quelques pratiques actuelles et propositions à considérer au regard du CECRL}

Evaluating French for Academic Purposes acquisition: current practices and proposals in regards to the CEFRL

Nathalie Gettliffe

\section{OpenEdition}

Journals

Édition électronique

URL : https://journals.openedition.org/apliut/3596

DOI : 10.4000/apliut.3596

ISSN : 2119-5242

Éditeur

APLIUT

Édition imprimée

Date de publication : 15 février 2013

Pagination : 114-129

ISSN : 2257-5405

Ce document vous est offert par Bibliothèque nationale et universitaire de Strasbourg (BNUS)

\section{bon}

\section{Référence électronique}

Nathalie Gettliffe, «Validation d'acquis en français sur objectif universitaire (FOU) : quelques pratiques actuelles et propositions à considérer au regard du CECRL », Recherche et pratiques pédagogiques en langues de spécialité [En ligne], Vol. XXXII Nº 1 | 2013, mis en ligne le 22 juillet 2014, consulté le 17 mai 2021. URL : http://journals.openedition.org/apliut/3596; DOI : https://doi.org/10.4000/apliut.3596 


\section{Nathalie Gettliffe}

\section{Université de Strasbourg}

Validation d'acquis en français sur objectif universitaire (FOU): quelques pratiques actuelles et propositions à considérer au regard du CECRL

\section{Mots-clés}

CECRL, certification en langue étrangère, français langue étrangère (FLE), français sur objectifs spécifiques (FOS), français sur objectif universitaire (FOU), langue sur objectifs spécifiques, LANSAD

\section{Résumé}

À l'heure de la mise en place d'une politique des langues commune pour le secteur Langues pour spécialistes d'autres disciplines (LANSAD) de l'Université de Strasbourg, la question des certifications est au centre des préoccupations. Quel niveau exiger en fin de parcours universitaire, et comment valider des niveaux de compétences en langue sur objectifs spécifiques tout en s'appuyant sur les principes du Cadre européen commun de référence pour les langues (CECRL)?

Notre regard se portera sur les mobilités entrantes et les dispositifs accompagnant les étudiants étrangers à l'Université de Strasbourg. Nous présenterons les résultats d'entretiens avec des intervenants en FOU concernant le contenu des parcours qu'ils proposent dans différentes disciplines et les validations s'appuyant sur des descripteurs du CECRL qui y sont associées.
Evaluating French for Academic Purposes acquisition: current practices and proposals in regards to the CEFRL

\section{Keywords}

CEFRL, second language certification, French as a Foreign Language, French for Specific Purposes, French for Academic Purposes, Language for Specific Purposes, language learning for non specialists

\section{Abstract}

The University of Strasbourg is currently engaged in setting common second language learning objectives for all its teaching departments. Language certifications are at the heart of all discussions. What should be the final language outcomes for students when finishing their degrees and, just as importantly, in the light of the CEFRL, what sets of practices should be adopted to validate competences in language for specific purposes?

Our study will focus on various French for Academic Purposes classes offered to foreign students enrolled at the University of Strasbourg. Interviews with heads and teachers of various language centers will outline the content of such classes and the CEFRL-based-evaluations available to foreign students. 


\section{Validation d'acquis en français sur objectif universitaire (FOU) Quelques pratiques actuelles et propositions à considérer au regard du CECRL}

\section{Problématique}

Alors que l'enseignement des langues pour spécialistes d'autres disciplines (LAN$\mathrm{SAD}$ ) existe depuis des décennies dans les universités françaises, la certification de ces enseignements est relativement récente avec la mise en place progressive du Certificat de compétences en langues pour l'enseignement supérieur (CLES). Bien que s'appuyant sur le domaine des études tel que proposé dans le Cadre européen commun de référence pour les langues (CECRL), cette certification reste cependant incomplète au vu des différences discursives qui peuvent exister entre les disciplines, et elle laisse donc peu de place à la validation de niveaux de compétences dans des cursus qui intègrent un enseignement en langue de spécialité. Ainsi, nous voyons apparaître une fracture entre des enseignements en langue plus ou moins générale, qui proposent des certifications nationales (CLES) ou internationales s'appuyant sur le CECRL, et des enseignements innovants en langue sur objectifs spécifiques qui, pour le moment, fonctionnent souvent sans certification ou avec des certifications locales qui se fondent rarement sur des descripteurs du CECRL.

Notre objectif est de présenter les pratiques actuelles en termes de certification en en français sur objectifs spécifiques (FOS) et en français sur objectif universitaire (FOU) au vu du CECRL. Notre propos s'attardera sur trois dispositifs d'enseignement du français langue étrangère (FLE) proposés à des étudiants nonfrancophones intégrant l'Université de Strasbourg, et plus précisément sur des dispositifs censés soutenir l'apprentissage du FOU ${ }^{1}$. Un bref rappel de l'historique

1 Une note de l'Association de didactique du Français langue étrangère (AsDiFLE) (http://www.asdifle.com/node/24) rend compte des débats qui existent parmi les didacticiens du FLE concernant la multiplication terminologique dans le domaine FLE. Au sein de l'Université de Strasbourg, certains cursus privilégient le français langue de spécialité et d'autres le FOS ou le FOU. Mangiante et Parpette (2004) définissent les enseignements en langue de spécialité comme répondant à une logique d'offre de la part de l'institution (ex : français des affaires proposé dans une Alliance française asiatique à des étudiants qui pensent éventuellement faire du commerce avec des pays francophones), alors que les enseignements sur objectifs spécifiques s'inscrivent dans une logique de demande de la part d'étudiants ou de travailleurs confrontés à un besoin urgent de maîtrise de la langue (ex : infirmières espagnoles embauchées dans un hôpital français). Le FOU est une déclinaison du FOS ; sa démarche s'appuie sur une ingénierie pédagogique particulière adaptée à un public adulte 
universitaire strasbourgeois permettra, dans un premier temps, de comprendre le contexte des formations analysées. Nous procéderons ensuite à une présentation des certifications existantes en langue générale et en langue sur objectifs spécifiques avant d'examiner les pratiques certificatives de chacun des trois centres de langues. Des propositions pédagogiques clôtureront notre propos.

\section{Contexte}

Depuis de nombreuses années, les universités strasbourgeoises (Marc Bloch, Robert Schuman et Louis Pasteur) se sont interrogées sur les modalités d'enseignement des langues pour spécialistes d'autres disciplines, et chacune a pu apporter des réponses plus ou moins concertées (cf. tableau 1 ci-dessous). L'Université Louis Pasteur fonctionne avec un service central (ULP Langues) qui gère plusieurs centres de ressources en langues installés au sein des différentes facultés scientifiques et organisés autour d'enseignants conseillers. Certains cours de sciences peuvent aussi être présentés en langue anglaise. Pour l'Université Marc Bloch et l'Université Robert Schuman, les enseignements de langue restent, pour la majorité, de type traditionnel (face-à-face) avec des enseignants professeurs agrégés (PRAG), certifiés (PRCE) ou des maîtres de langue. Un centre de ressources et d'apprentissage en langue (CRAL) existe aussi dans l'Université Marc Bloch et propose des formations en mode hybride (présentiel/distanciel) aux différentes facultés. Une école des langues et des cultures (SPIRAL) ainsi qu'un centre de formation en langues (CFL) ouvre ses portes à des acteurs non-universitaires (diplomates, militaires, etc.). Finalement, l'Institut international d'études françaises (IIEF) se spécialise dans l'enseignement du FLE pour des apprenants adultes souhaitant ensuite suivre des cursus universitaires.

Tableau 1. Récapitulatif des dispositifs d'enseignement des langues dans les anciennes universités strasbourgeoises

\begin{tabular}{|l|l|}
\hline Université Louis Pasteur & 8 centres de ressources (anglais, allemand, espagnol, FLE) \\
\hline \multirow{3}{*}{ Université Marc Bloch } & $\begin{array}{l}\text { Cours en présentiel (25 langues) } \\
\text { Maison des langues et des cultures (26 langues en autono- } \\
\text { mie guidée) } \\
\text { Centre de formation en langues (anglais, allemand en pré- } \\
\text { sentiel) } \\
\text { Centre de ressources et d'apprentissage en langues (anglais, } \\
\text { allemand en mode hybride }\end{array}$ \\
\hline Université Robert Schuman & Cours en présentiel (anglais, allemand, espagnol) \\
\hline $\begin{array}{l}\text { Institut international } \\
\text { d'études françaises (IIEF) }\end{array}$ & Cours en présentiel FLE (niveaux A1 à C2) \\
\hline
\end{tabular}

étudiant et mobilisant fortement les analyses discursives et les documents authentiques. Le concept de FOU recoupe parfois celui de langue de spécialité, comme dans le cadre des enseignements à l'Institut international d'études françaises (IIEF). 
La fusion des trois universités sous le nom Université de Strasbourg (UDS) en 2009 a posé la question d'une politique des langues commune pour l'ensemble du nouveau site universitaire avec, pour le moment, des offres de formation toujours très disparates selon les facultés, tant du point de vue des finalités que des pratiques pédagogiques. Un rapport appelé Ham a détaillé de manière précise les différentes formations proposées et, début 2012, plusieurs groupes de travail ont élaboré des propositions concernant des points particuliers afin d'impulser une ligne directrice au projet «UDS Langues » (certifications, place de l'anglais, FLE, langues et mobilités, formation continue, multilinguisme).

Les certifications représentent un enjeu important pour l'Université de Strasbourg car elles valident non seulement des niveaux en langue, mais elles peuvent aussi orienter les enseignements de langue. Par exemple, l'hégémonie de l'anglais peut être remise en cause en valorisant, par le biais de certifications multiples, les compétences multilingues; une augmentation de l'offre de cours sur et en langues de spécialité pour favoriser les mobilités estudiantines pourrait être impulsée si les étudiants étaient orientés vers des certifications en langue de spécialité ; les démarches d'enseignement de type actionnel pourraient être généralisées à l'ensemble du site si le Certificat en langues pour l'enseignement supérieur (CLES) était imposé comme un premier pré-requis pour tous les étudiants de licence. Comme le résument Noël-Jothy et Sampsonis (2006: 12), « au plan pédagogique, l'évaluation agit comme un levier puissant sur l'enseignement ».

Avant de continuer avec la description des pratiques actuelles de certifications à l'Université de Strasbourg, tournons-nous rapidement vers quelques définitions et concepts prenant en compte certifications et CECRL.

\section{Certifications}

\subsection{Certifications générales et CECRL}

La finalité de l'évaluation certificative est la délivrance d'un diplôme reconnu socialement et professionnellement (Noël-Jothy \& Sampsonis 2006 : 11). Adossé aux échelles du CECRL, le processus de certification permet de mesurer toutes les compétences langagières (production, réception et interaction) pour un niveau donné (Hardy 2006). La certification diffère donc du test, outil d'information qui peut renseigner sur plusieurs niveaux de la compétence communicative (exemple : A1 en production orale continue et $\mathrm{B} 1$ en compréhension écrite). Le CLES, comme le diplôme d'études élémentaires en langue française (DELF), sont des certifications, alors que le Test of English as a Foreign Language (TOEFL) ou le Test de connaissances en français (TCF) sont, comme leur nom l'indique, des tests au sens de la définition énoncée ci-dessus. Les activités proposées dans les certifications sont de natures diverses. Par exemple, le CLES est organisé autour de la réalisation d'une tâche finale mobilisant les expressions orales et écrites au sein d'un scénario de communication. Toutefois, dans cette même certification, des micro-tâches de 
compréhension orale et écrite sont présentées sous forme de questions à choix multiples et de réponses courtes, activités plus proches des outils d'évaluation traditionnels comme, par exemple, les examens de Cambridge. Pour ce qui est des descripteurs du CECRL mobilisés pour évaluer les niveaux, ils sont issus traditionnellement du domaine éducationnel (voir, par exemple, CLES 1, CLES 2), mais d'autres certifications (DELF, DALF, Cambridge English, DCL...) convoquent les domaines public, personnel et professionnel ${ }^{2}$.

\subsection{Certifications en langue sur objectifs spécifiques et CECRL}

La réflexion sur les certifications en langue sur objectifs spécifiques est relativement récente en France (Hardy 2006) même si, dans les faits, certaines certifications sont déjà inscrites dans le paysage depuis plus longtemps (Cambridge Business, Diplôme de compétence en langues, DELF Pro...). L'objectif de ces dernières est d'attester des savoir-faire caractérisant la mise en œuvre de la compétence en langue en milieu professionnel et/ou universitaire. En effet, il s'agit de s'assurer, dans le cadre des mobilités étudiante et professionnelle, que l'étudiant pourra suivre des cours de sa spécialité en langue étrangère et qu'il pourra aussi effectuer des stages ou démarrer une carrière internationale dans un pays non-francophone.

L'adossement de l'enseignement des langues sur objectifs spécifiques au CECRL reste complexe (Fries-Verdeil 2009) car, si le CECRL permet de s'appuyer sur les domaines professionnel et éducationnel pour proposer des tâches sociales, les descripteurs de ces deux champs restent relativement généraux. Par exemple, le descripteur suivant pour un niveau B2, «EST CAPABLE DE parcourir un texte pour retrouver l'information pertinente et d'en saisir l'essentiel» (Conseil de l'Europe 2001 : 183), ne permet pas de rendre compte des spécificités des discours circulant dans les différentes disciplines.

En revanche, bien que parfois réduit à un seul but d'évaluation en langue générale, le CECRL propose plusieurs pistes pour l'organisation d'une certification en langue sur objectifs spécifiques. Les programmes d'apprentissage et les certifications peuvent être :

- $\quad[. .$.$] modulaires, développant les compétences de l'apprenant dans un secteur$ limité pour un objectif bien déterminé,

- $[\ldots]$ partiels, ne prenant en charge que certaines activités et habilités (la réception, par exemple) et laissant les autres de côté (Conseil de l'Europe 2001 : 12).

On voit ici tout l'intérêt de ces propositions didactiques qui montrent la flexibilité du CECRL pour des certifications en langue sur objectifs spécifiques pouvant reposer sur des validations de compétences partielles (par exemple, seules la com-

\footnotetext{
${ }^{2}$ Quelques exemples de catégories situationnelles telles que résumées dans le CECRL (2001: 43): billets, menus, associations... (domaine public), famille, décoration, vacances... (domaine personnel), réunions, hôtels, note de rapport... (domaine professionnel).
} 
préhension et l'expression orales seront évaluées) à l'aide de tâches très ciblées. Le cas de l'enseignement des langues sur objectifs spécifiques est par ailleurs nommément évoqué, bien que rapidement, dans le chapitre 6 :

\begin{abstract}
Les objectifs de l'enseignement/apprentissage peuvent en effet être conçus en termes variés [...] [e]n termes d'insertion fonctionnelle optimale dans un domaine particulier (voir Section 4.1.1) et relever alors du domaine public, ou du domaine professionnel, ou du domaine éducationnel ou du domaine personnel. Il est des cas où l'apprentissage d'une langue étrangère a pour objectif essentiel une meilleure adéquation à un poste de travail ou à un contexte de formation ou à certaines conditions de vie quotidienne dans un pays étranger. Comme pour les autres constituants majeurs du modèle présenté, de telles visées donnent lieu à des affichages explicites, notamment «d'objectifs spécifiques», de « cours de spécialité », de «langues des professions ", de "préparation au séjour à l'étranger », "d'accueil linguistique de travailleurs migrants ». Ce qui ne veut pas dire que la prise en compte de besoins particuliers d'un public déterminé ayant à accommoder sa compétence plurilingue et pluriculturelle à un domaine précis d'activité sociale s'accompagne toujours d'un traitement pédagogique adéquat à cette visée. (Conseil de l'Europe 2001 : 106-107; caractères gras dans le texte)
\end{abstract}

Nous allons maintenant décrire les pratiques de certification et d'évaluation concernant l'accueil des étudiants étrangers de l'Université de Strasbourg dans des dispositifs de formation en FOU, ceci afin d'apprécier les propositions actuelles au regard des directions impulsées par le CECRL. Les dispositifs décrits sont les plus aboutis en termes d'enseignement de langue sur objectifs spécifiques ${ }^{3}$, beaucoup de facultés se contentant de faire de la remise à niveau générale. Des entretiens avec chacun des responsables de formation nous ont permis de préciser les pratiques et les évolutions possibles.

\title{
4. Dispositifs d'enseignement du FOU
}

Au sein de l'Université de Strasbourg, il existe des cursus qui privilégient le français langue de spécialité (l'Institut international d'études françaises) et d'autres qui privilégient le FOU (le Centre de ressources en langue FLE et le Département de didactique des langues).

\subsection{L'Institut international d'études françaises (IIEF)}

L'Université de Strasbourg accueille des étudiants étrangers au sein de plusieurs dispositifs. Les plus prévoyants s'orientent plusieurs semaines avant leur intégration vers l'Institut international d'études françaises (IIEF), spécialisé depuis 1919 dans les formations FLE. Les parcours de niveau plus élevé comme le diplôme d'études françaises (niveau B2), le diplôme avancé d'études françaises (niveau C1) et le diplôme supérieur d'études françaises (niveau C2) s'appuient sur des cours en

\footnotetext{
${ }^{3}$ Pour une approche plus détaillée des contenus pour l'enseignement des langues sur objectifs spécifiques, consulter Lerat (1995), Lehmann (1993), Hyland (2002), Belcher (2009), Mangiante \& Parpette $(2004 ; 2011)$.
} 
présentiel d'un volume horaire d'environ 18 heures par semaine. Les apprenants sont considérés comme étudiants au niveau de la prise en charge administrative (inscription à l'université, couverture médicale, allocation logement, etc.) et ils suivent des parcours qui doivent leur permettre d'intégrer leur spécialité d'origine. À ce titre, les enseignements sont répartis entre modules de formation linguistique (une dizaine d'heures) et modules de formation «culturelle» incluant des cours très variés comme "Le français du luxe et de l'art», "Les institutions politiques françaises », «Les genres de la peinture », «La philosophie en France », « Quel français pour enseigner les sciences? », etc. Des modules en immersion sont aussi proposés, c'est-à-dire que l'étudiant a la possibilité de s'inscrire dans un ou plusieurs cours auprès de la faculté de son choix : il pourra ainsi appréhender les exigences linguistiques et disciplinaires pour les études qu'il envisage de faire.

La plaquette de présentation de ces diplômes affiche les modalités de contrôle des différents enseignements. Chaque compétence (compréhension orale, compréhension écrite, production orale, production écrite) est évaluée par le biais d'un contrôle continu et d'un examen terminal afin de mettre l'apprenant dans le rythme classique des évaluations à l'université. Une certification locale ${ }^{4}$ est délivrée sous forme d'un relevé de notes pour chacune des unités d'enseignement suivies. On remarque là encore l'empreinte du système universitaire quant au format du relevé de cette évaluation certificative. Pour autant, cette dernière se différencie d'une juxtaposition d'évaluations sommatives dans le sens où chaque compétence langagière est évaluée selon les descripteurs d'un niveau du CECRL, ce qui lui donne une valeur dépassant le simple cadre de l'IIEF.

Un entretien avec la directrice de l'IIEF confirme, dans un premier temps, que les enseignements sont organisés en fonction des niveaux du CECRL. Un test de placement positionne l'apprenant en début de parcours et un niveau final est visé, atteignable en un ou deux semestres. Si les compétences générales sont adossées au CECRL, les apprentissages en langue sur objectifs spécifiques ne sont évalués que sur les contenus (content-based learning). Aucune évaluation linguistique (focus on form) n'est retenue pour les langues de spécialité et les descripteurs du CECRL ne sont nullement convoqués. Les domaines du CECRL «Études» et «Travail» (pourtant sous-jacents à plusieurs cours) ne sont inscrits nulle part. Seules les notes apparaissent sur le relevé, et aucun indicateur des compétences acquises n'y est ajouté. Pour le moment, aucune réflexion n'est engagée quant à des approches du type Portfolio européen des langues (PEL) pour valider les modules en immersion ou les modules en langue de spécialité. Quelques initiatives de certification existent de manière ponctuelle, les étudiants canadiens et américains se souciant de la transférabilité des unités d'enseignement strasbourgeois en crédits nord-américains.

\footnotetext{
${ }^{4}$ L'institut international d'études françaises délivre les certifications suivantes : Certificat de français (A1), Certificat de français (A2), Certificat pratique de langue française (B1), Diplôme d'études françaises (B2), Diplôme avancé d'études françaises (C1) et Diplôme supérieur d'études françaises (C2).
} 
Toutefois, la ré-écriture des descriptifs de cours locaux en «syllabi» nordaméricains ne s'appuie en aucun cas sur les descripteurs du CECRL, peu usités en Amérique du Nord.

\subsection{Centre de ressources en Français langue étrangère (FLE)}

Le deuxième centre d'accueil des étudiants étrangers se situe au sein de l'ex-ULP Langues. Un centre de langues et de ressources FLE (CRL FLE) leur est dédié avec, pour la plupart des étudiants scientifiques, la possibilité de suivre un parcours intégré de plusieurs heures par semaine alliant travail individuel, regroupements et séances conseil. Les ressources en accès libre (papier et en ligne) sont aussi disponibles pour les étudiants venant d'autres facultés, notamment de l'ex-Université Marc Bloch et de l'ex-Université Robert Schuman. Des ateliers linguistiques (cafés linguistiques trilingues, phonétique, conversation...) sont aussi proposés à tous les étudiants pour travailler de manière plus ou moins informelle des points de langue. La particularité de ce centre de langues est de ne pas regrouper les étudiants par niveaux et de se focaliser sur les difficultés des étudiants en FLE, tant sur le plan général que sur des aspects linguistiques spécifiques à leur discipline.

L'évaluation des étudiants est dite «continuée » et se décline en trois temps : une évaluation intermédiaire qui porte sur le fonctionnement de l'étudiant dans le centre, une évaluation "au fil de l'eau » (un écrit ou un oral adapté au niveau de l'étudiant) et une évaluation finale (la soutenance d'un dossier sur le travail réalisé en autonomie). Aucune certification n'est proposée aux étudiants par le centre.

L'entretien avec la directrice du centre confirme qu'une certification en FOU n'est pas envisagée pour l'instant. En revanche, plusieurs pratiques d'étudiants dans ce centre montrent que, d'une part, l'apprentissage du FOU forme une part importante de l'activité du centre, et que, d'autre part, les différentes disciplines créent des besoins variés. Tout d'abord, la directrice du centre convient qu'un « vent de panique » souffle sur les étudiants pendant les premières semaines de cours et que ces derniers se précipitent sur les manuels spécialisés tant en droit qu'en économie, en sciences ou en affaires, afin de pallier un déficit terminologique évident. Un important travail sur la compréhension de l'écrit est fourni par les étudiants pour presque toutes les disciplines, sauf par ceux issus de la faculté de mathématiques, qui travaillent sur l'expression orale en français des concepts mathématiques qu'ils sont tout à fait capables de comprendre lors de démonstrations au tableau. Les étudiants en psychologie, passées les premières semaines de cours, se focalisent sur la compréhension des consignes afin de pouvoir répondre aux nombreux QCM qui jalonnent leur parcours de formation. Les étudiants en droit sont confrontés à une langue de spécialité très marquée, mais la méthodologie universitaire centrée sur l'apprentissage par cœur permet à des étudiants non-francophones de réussir cette formation sans passer par un travail approfondi autour de l'expression écrite, à tel point que certains auront une compétence en langue juridique très élevée tout en ayant des difficultés à pouvoir fonctionner en français langue générale. 
À cette première phase marquée par un travail centré sur les langues des disciplines succède une période plus ou moins longue qui permet aux étudiants de reprendre et d'approfondir des compétences langagières en français général. D'ailleurs, la directrice note que le dossier d'évaluation finale proposé par les étudiants porte surtout sur cet aspect de l'apprentissage. Les étudiants peuvent décider de quitter le CRL FLE pour se diriger vers l'étude d'autres langues s'ils le souhaitent, mais ils reviennent souvent en candidats libres lorsque d'autres obstacles se présentent, comme la rédaction d'un mémoire ou d'un doctorat. À ce sujet, la directrice observe qu'il est parfois difficile pour les enseignants du CRL FLE d'aider les étudiants issus de disciplines scientifiques avec des structures de discours et des terminologies très éloignées du français usuel.

Bien que la biographie langagière du PEL soit renseignée par les étudiants lors de leur accueil dans le CRL FLE, les autres composantes (passeport des langues et dossier) ne sont pas utilisées car le PEL en version certificative ${ }^{5}$ serait, selon la directrice, trop lourd à gérer. Tout le travail réalisé au CRL FLE est donc soumis à des notes qui ne sont pas accompagnées de descripteurs reposant sur le CECRL. Pour la directrice, réussir les examens de sa spécialité équivaut à une certification informelle.

\subsection{Département de didactique des langues et du FLE}

À l'Université Marc Bloch (Lettres, langues et sciences humaines), un dispositif central avait été aussi mis en place avant la fusion des universités et portait sur une aide personnalisée en méthodologie et en rédaction de travaux universitaires pour les étudiants non-francophones. Il reste maintenant des unités d'enseignement FLE assurées par les différentes facultés selon leurs moyens financiers. C'est le cas au département de didactique des langues et du FLE où deux unités d'enseignement en FOU sont imposées aux étudiants de Master n'ayant pas acquis certaines bases méthodologiques et linguistiques (bien qu'un niveau B2 soit requis à l'entrée du diplôme). Au total, ce sont 48 heures $^{6}$ de cours annuelles qui portent sur la remédiation linguistique, la méthodologie universitaire, la compréhension de textes de la discipline, la prise de notes sur les discours oraux et écrits de la discipline, la compréhension de consignes d'examen, l'expression écrite pour des écrits courts et longs (mémoire) et l'expression orale pour des exposés ou pour une soutenance de mémoire. L'évaluation s'articule, pour le premier semestre, autour de travaux d'expression écrite, d'un devoir écrit de compréhension orale, et d'expression écrite sur des discours didactiques. Pour le second semestre, une présentation orale de la problématique de recherche des étudiants constitue le support de la première

\footnotetext{
${ }^{5}$ Notons que le but du dossier du PEL n'est pas d'être un support pour une évaluation certificative mais de proposer à l'apprenant une modalité d'évaluation formative (Frath 2011).

${ }^{6}$ Les étudiants ayant un niveau de compétence en FLE élevé (C2) sont invités à suivre des enseignements en langues (autres que leur langue maternelle) pour l'unité d'enseignement Langues inscrites en semestre 1, 2 et 3 de la maquette du Master en didactique des langues et du FLE.
} 
évaluation, accompagnée de la rédaction d'une synthèse à partir de plusieurs articles de recherche. Les évaluations continues ne s'appuient sur aucun descripteur du CECRL et aucune certification n'est encouragée ou proposée.

L'entretien avec l'une des enseignantes de ce module révèle que ces modules de FOU sont récents car la configuration initiale de cette aide était plutôt centrée sur du français général. Différentes formules ont été testées au cours des trois dernières années afin d'articuler au plus près la formation en FOU et les enseignements en didactique des langues. Par le biais d'entretiens entre le responsable de ce module et les enseignants des disciplines, le contenu actuel du cours s'est dessiné tout en restant flexible pour accueillir des étudiants avec des niveaux variés dans les différentes compétences. Une certification locale ${ }^{7}$ pourrait être envisagée à l'avenir par le biais de portfolios centrés sur les dossiers réalisés pour les différents cours, mais il resterait un travail important de définition des descripteurs à faire.

\section{Discussion}

\subsection{Pratiques de certification en FOU à l'UDS et adossement au CECRL}

$\mathrm{Si}$, de manière générale, au sein de l'Université de Strasbourg, des certifications en langue générale sont proposées (comme le CLES) ou imposées (par ex., le $\mathrm{TOEIC}^{8}$ ) dans le cadre d'unités d'enseignement ou hors de ce cadre, les pratiques actuelles en termes de certifications en FOU sont quasiment inexistantes. Elles se limitent au mieux à des diplômes internes agrémentés d'un relevé de notes qui ne fait pas état des contenus linguistiques travaillés en regard des contenus de spécialité. Les enseignants et les directrices de dispositifs ne distinguent pas les compétences générales nécessaires pour réussir un examen ou des travaux dans une discipline (mémorisation, organisation, rédaction, compréhension...), et les compétences linguistiques mobilisées pour réaliser ces mêmes productions. On peut facilement imaginer qu'un étudiant échoue à un examen alors qu'il maîtrise très bien la langue de spécialité (c'est par exemple le cas de nombreux francophones). À l'inverse, réussir une unité d'enseignement n'atteste pas non plus d'une maîtrise des variétés discursives de la discipline. En effet, des travaux de groupes, des compensations entre unités d'enseignement, une notation assez large de certains enseignants permettent à des étudiants faibles en langue de spécialité de valider leur semestre. La composante langue est souvent un facteur essentiel dans la réussite des études des non-francophones, mais elle n'est pas la seule. On remarque par ailleurs qu'aucun processus d'évaluation linguistique n'existe pour les cours suivis en immersion.

\footnotetext{
${ }^{7}$ Pour un exemple de certification locale adossée au CECRL, voir Frath 2012.

${ }^{8}$ Le TOEIC est présenté comme une certification en langue de spécialité mais, au vu des épreuves proposées, on peut questionner cette spécificité.
} 
Pour ce qui est de l'adossement des évaluations aux échelons du CECRL, il est présent dans les structures qui privilégient les groupes de niveaux (IIEF, département de didactique des langues). Toutefois, les échelons sont mentionnés dans leur globalité et aucun descripteur n'est précisé dans le cadre des évaluations continues.

Le CECRL propose aussi des modalités d'enseignement et d'évaluation centrés sur des tâches globales ${ }^{9}$; cependant, aucun des dispositifs examinés ne s'articule autour de l'approche actionnelle, même si des activités comme "présenter oralement une problématique de recherche» s'y apparentent. Finalement, l'utilisation de portfolios n'est mobilisée que lorsque la biographie langagière est concernée. Au final, non seulement la certification en langue de spécialité est absente dans les pratiques actuelles de l'Université de Strasbourg mais, de surcroît, les enseignements et les évaluations proposés convoquent peu les éléments clés (et souvent innovants) du CECRL ${ }^{10}$.

\subsection{Propositions}

Cela fait à peine une dizaine d'années que les linguistes (Boch \& Grossman 2001 ; Rinck 2004 ; Delcambre \& Reuter 2002) et les didacticiens (Mangiante \& Parpette 2011 ; Bouchard \& Parpette 2007 ; Goes \& Mangiante 2007 ; Fougerouse 2010) en France s'intéressent aux littéracies universitaires pour les étudiants français et au FOU pour les étudiants étrangers alors que ces domaines (Composition Studies et Academic English) sont étudiés de manière intense depuis plus de vingt ans par les chercheurs anglo-saxons (par exemple, Swales 1990 ; Hyland 2000). En conséquence, peu de recherches et de retours d'expérience ont été publiés concernant la mise en place de manière généralisée de certifications en langue sur objectifs spécifiques, tant pour les mobilités sortantes (étudiants natifs s'inscrivant dans des universités étrangères) que pour les mobilités entrantes (étudiants étrangers s'inscrivant dans des universités françaises). En France, le CLES, qui date d'une dizaine d'années, a contribué à développer la certification en langue générale dans l'enseignement supérieur mais, étant donné la lourdeur du système (une centralisation complexe et des descripteurs critiqués pour leur manque de fiabilité), on peut se demander s'il est raisonnable d'espérer un jour posséder une panoplie d'évaluations permettant de certifier des compétences en langue de spécialité.

\footnotetext{
${ }^{9}$ Dans le domaine qui nous intéresse (éducationnel), on peut imaginer des tâches globales du type « rédiger une fiche bibliographique », « élaborer une note de synthèse », « s'inscrire dans une option libre », « dessiner un plan de fouilles archéologiques » qui seraient plus en phase avec des activités réalisées au sein des différents cursus universitaires.

${ }^{10}$ Organisation des enseignements/apprentissages autour de tâches globales, modularité des enseignements, acquisition de compétences partielles plurilingues, centration sur le domaine des études, mobilisation d'outils souples pour des auto-évaluations (PEL), élaboration de descripteurs de compétences pour des langues de spécialité, développement de compétences transversales d'apprentissage (savoirs, savoir-faire, savoir-être, savoir-apprendre...) et de stratégies communicationnelles.
} 
Il faudrait certainement, dans un premier temps, proposer aux étudiants les certifications existantes. En FLE, le Centre international d'études pédagogiques (CIEP) a élaboré un Diplôme élémentaire en langue française pour les professionnels (DELF Pro) qui propose tous les niveaux du CECRL ; le Ministère de l'éducation nationale a aussi travaillé à l'élaboration d'un diplôme de compétence en langues (DCL) qui vise à certifier des compétences pour le milieu du travail ; la Chambre de commerce et d'industrie de Paris (CCIP) offre des certifications en français des affaires (3 niveaux), en français juridique, et en français scientifique et technique pouvant intéresser les écoles de management et les facultés juridiques et scientifiques; enfin, l'Alliance française de Paris propose un diplôme des hautes études françaises niveau C2 (option littérature ou traduction) qui pourrait être adopté par des facultés de Lettres ou de Langues étrangères. Ces certifications diverses aideraient les étudiants étrangers à mettre en avant leurs acquis en langue française auprès d'employeurs dans leurs pays respectifs.

Pour une offre plus locale et moins onéreuse, plusieurs pistes sont à envisager. Tout d'abord, il serait important dans un premier temps de répertorier et d'analyser les besoins des différentes disciplines et des divers métiers approchés lors de stages professionnels. Cependant, malgré une approche relativement bien éprouvée (Mangiante \& Parpette 2004), de nombreux discours restent à analyser. Une autre approche consiste, comme le proposent encore Mangiante et Parpette (2011: 124) à établir une liste de macro-compétences communes à toutes les disciplines afin de les mettre en regard des descripteurs du CECRL. Ainsi, à partir d'une typologie des productions écrites universitaires (restitutions de cours, commentaires et synthèses, études de cas et simulations), il est possible de rédiger un référentiel transversal établissant une progression entre les niveaux A (reproduire, identifier et recopier), $\mathrm{B}$ (reformuler, étendre, diversifier) et C (relier, apparier, créer). Une carte de compétences pour l'intégration universitaire est effectivement proposée par ces auteurs avec un adossement au niveau du CECRL (voir figure 1). 
Figure 1. Carte de compétences pour l'intégration universitaire adossée au CECRL

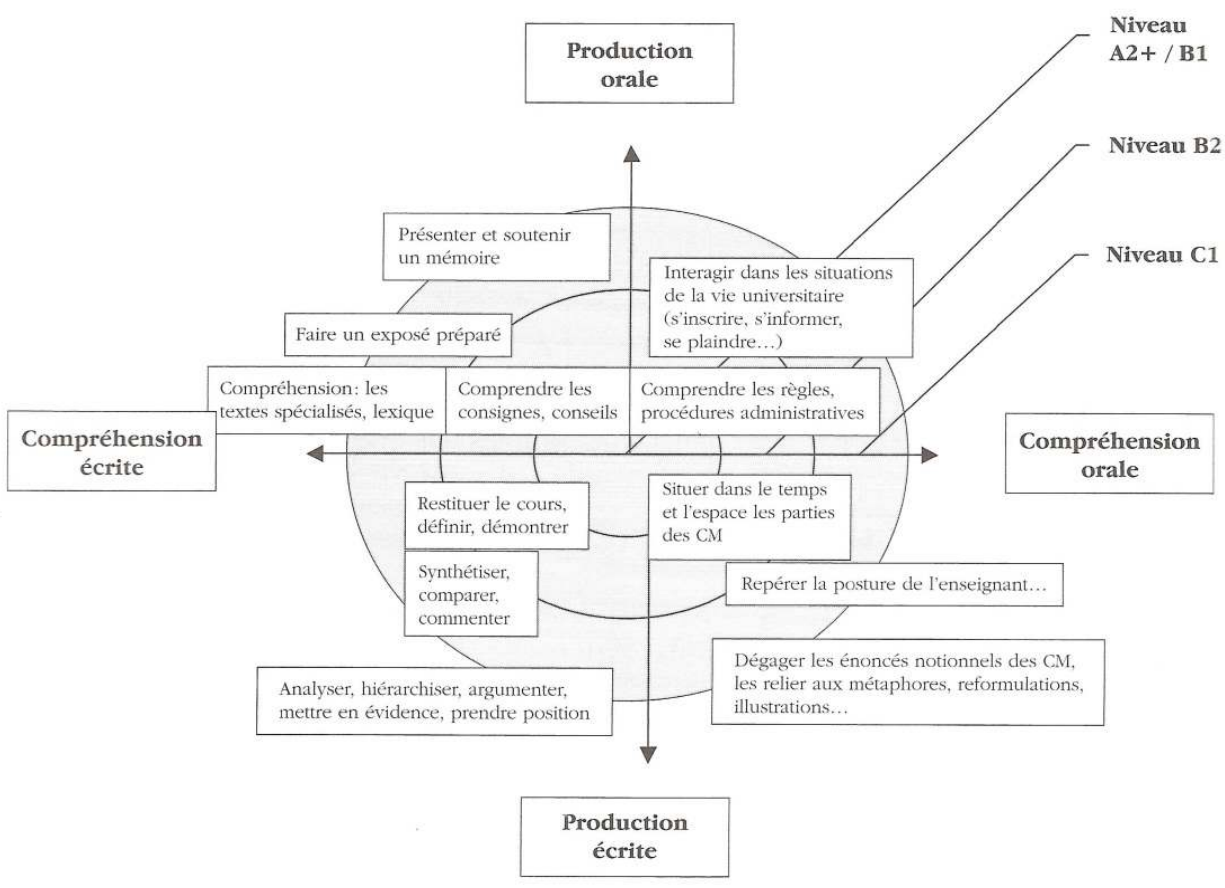

(Mangiante \& Parpette $2011: 214$ )

Une autre solution consiste à choisir d'évaluer des compétences partielles en s'appuyant sur des descripteurs du CECRL déjà éprouvés, notamment dans le domaine des études. Ainsi, Fries-Verdeil (2009) a tenté avec succès de pallier la « sous-conceptualisation fondamentale de la langue de spécialité » (Petit $2006: 29$ ) dans le CECRL en n'évaluant que trois compétences de niveau B2 dans un dispositif d'anglais de spécialité pour étudiants de Master scientifique. Les compétences visées (expression orale, compréhension écrite et expression écrite) sont travaillées et évaluées au moyen d'une tâche finale qui consiste en une présentation orale d'une recherche scientifique de leur choix et une prise de notes des exposés oraux des autres étudiants, en vue de rédiger un résumé. Cette évaluation est complétée par des séances de prises de notes sur des vidéos regardées en cours.

Pour une certification locale, il est aussi possible de s'appuyer sur les PEL. Cependant, comme le note Methy (2005) lors de son expérimentation auprès de 569 étudiants, peu d'entre eux $(17 \%)$ utilisent le PEL de manière régulière, même si plus de la moitié des étudiants le trouvent intéressant et qu'un quart s'en servent pour se fixer des objectifs. Lourd à gérer dans des situations en autonomie, le PEL permet toutefois d'accompagner des étudiants en situation d'intégration en adaptant certains descripteurs et en valorisant des productions consignées dans la partie 
dossier et ce, particulièrement pour toutes les activités réalisées dans un contexte d'immersion. Si l'enseignant n'est pas toujours disponible pour accompagner l'étudiant dans sa démarche d'évaluation, une co-évaluation entre pairs (Springer 2009) est aussi envisageable.

S'il est un domaine où langue sur objectifs spécifiques et CECRL se rejoignent, c'est certainement la perspective actionnelle. Toute certification locale devrait donc se fonder sur des tâches globales authentiques, miroir d'activités réalisées dans les enseignements disciplinaires. Il est clair que réaliser un exposé en classe de FOU ou préparer en classe de FOU un exposé qui sera présenté dans son contexte disciplinaire consacre l'étudiant dans son rôle d'acteur social. Un dispositif de filmage permettrait à l'enseignant de langue d'évaluer la performance de l'étudiant in situ.

Finalement, les compétences culturelles qui pour le moment n'apparaissent que marginalement dans les grilles d'évaluation (Huver 2012) pourraient être aussi travaillées afin que les étudiants prennent conscience du monde d'où ils viennent et du monde de la communauté cible (Conseil de l'Europe 2001 : 83). Aucune certification n'est pour le moment proposée dans ce domaine même si des formations existantes travaillent sur l'acquisition de ces compétences (voir Cabau 2012, sur des étudiants hongkongais inscrits en sciences économiques et sociales en partance pour la France ; Peyrard-Zumbihl 2008, sur des étudiants en IUP de gestion intégrant un stage en entreprise à l'étranger de six mois). Le projet NOVATRIS, porté par l'Université de Haute-Alsace, est doté en 2012 d'un budget de 4.5 millions d'euros dans le cadre de la réalisation d'un centre de compétences transfrontalières et devrait se pencher plus précisément sur ce sujet afin de réaliser son objectif, à savoir former des cadres triculturels pour l'espace rhénan.

\section{Conclusion}

En conclusion, comme nous l'avons déroulé dans nos propositions, une validation des acquis en langue de spécialité s'appuyant sur le CECRL est envisageable. Les certifications peuvent être proposées par des organismes extérieurs ou construites localement afin de mettre en adéquation totale les contenus d'enseignement et les évaluations certificatives. Les pratiques actuelles d'évaluation en FOU à l'Université de Strasbourg gagneraient à s'appuyer sur des outils proposés par le CECRL, comme par exemple le PEL, en intégrant des descripteurs transversaux aux différentes disciplines. Le dossier du PEL organisé autour de tâches réalisées dans le cadre de cours de FOU ou dans le cadre de cours en immersion permettrait aussi d'intégrer une perspective actionnelle dans les enseignements des langues de spécialité, de même qu'il permettrait de moduler le contenu des apprentissages/enseignements en valorisant des travaux réalisés en autonomie. Pour ce qui est des compétences interculturelles en langue de spécialité (notamment concernant la 
culture professionnelle), elles n'ont pas encore fait l'objet d'un référentiel en FOU et restent difficile à valider en l'état des recherches.

Afin de parfaire notre étude, nous nous proposons, dans une recherche ultérieure, de recenser les pratiques certificatives pour les mobilités sortantes afin d'identifier des pratiques innovantes intégrant le CECRL et d'apprécier aussi la part des apprentissages informels (Sockett \& Kusyk 2012) qui devraient sous-tendre l'acquisition des langues de spécialité au vue de la diffusion de plus en plus importante de contenus de cours en ligne.

\section{Bibliographie}

Belcher, D. 2009. English for Specific Purposes in Theory and Practice. Ann Arbor: Michigan ELT.

Boch, F. \& F. Grossman. (eds). 2001. Lidil, vol. 24. « Apprendre à citer le discours d'autrui ».

Bouchard, R. \& Ch. Parpette. 2007. «Reformulation et coformulation dans la communication scientifique avec support écrit ». In Schuwer, M., M.-C. Le Bot $\&$ E. Richard (eds). Pragmatique de la reformulation: types de discours, interactions didactiques. Rennes : Presses Universitaires de Rennes, 55-74.

Cabau, B. 2012. "Évaluation de compétences dans un programme universitaire hongkongais ». Langues modernes, vol. $1: 50-55$.

Conseil de l'Europe. 2001. Cadre européen commun de référence pour les langues : apprendre, enseigner, évaluer. Paris : Didier.

Delcambre I. \& Y. Reuter. 2002. «Images du scripteur et rapports à l'écriture». Pratiques, vol. 113-114: 7-28.

Fougerouse, M.-C. (ed.). 2010. L'évaluation des productions complexes en français langue étrangère/seconde dans l'enseignement supérieur. Paris: L'Harmattan.

Frath, P. 2011. « Le portfolio européen des langues et le cadre européen commun de référence: entre normalisation institutionnelle et responsabilité individuelle ». In Sachot, M. \& O. Schneider-Mizony (eds). Normes et normativité en éducation. Entre tradition et rupture. Paris : L'Harmattan, 71-93.

Frath, P. 2012. «Évaluation des étudiants non-spécialistes en langues à l'aide du CECRL ». Les langues modernes, vol. $1: 56-64$.

Fries-Verdeil, M.-H. 2009. « Mise en cohérence de l'anglais de spécialité et du CECRL en France : difficultés et enjeux ». Asp, vol. $56: 105-125$.

Goes, J. \& J.-M. Mangiante (dir.). 2007. L'accueil des étudiants étrangers dans les universités francophones. Arras : Artois Presses Universités.

Hardy, M. 2006. «Quelques réflexions sur le choix d'une certification à la suite du $\mathrm{XXVIII}^{\mathrm{e}}$ Congrès de l'APLIUT ». Recherche et pratiques pédagogiques en langues de spécialité, vol. $25, n^{\circ} 1$, http://apliut.revues.org/2554 (cons. le 16 juillet 2012). 
Huver, E. 2012. "Descripteurs et standard vs représentations et pluralité ». Les Langues modernes, vol. $1: 18-23$.

Hyland, K. 2000. Disciplinary discourses: Social interaction in academic genres. London : Longman.

Hyland, K. 2002. « Specificity revisited: how far should we go now? ». English for Specific Purposes, vol. $21: 385-395$.

Lehmann, D. 1993. Objectifs spécifiques en langue étrangère. Paris: Hachette FLE.

Lerat, P. 1995. Les langues spécialisées. Paris: PUF.

Mangiante, J.-M. \& Ch. Parpette. 2004. Le français sur objectif spécifique: de l'analyse des besoins à l'élaboration d'un cours. Paris : Hachette FLE.

Mangiante, J.-M. \& Ch. Parpette. 2011. Le français sur objectifs universitaires. Grenoble : PUG.

Methy, A.-M. 2005. «L'impact de l'utilisation du Portfolio européen des langues en cycle de Licence de sciences économiques ». Asp, vol. 47-48: 103-120.

Noel-Jothy, F. \& B. Sampsonis. 2006. Certifications et outils d'évaluation en FLE. Paris : Hachette FLE.

Petit, M. 2006. «Les descripteurs du cadre : quelle conception de la langue de spécialité ? ». In Haramboure, F. et al. (dir). Travaux des journées 2006 de l'EA 2025. Bordeaux : Université Victor Segalen Bordeaux 2, 14-29.

Peyrard-Zumbihl, H. 2008. «Langue de spécialité et didactisation de l'acquisition de la compétence de médiation culturelle en milieu universitaire par l'apprentissage expérientiel ». Les cahiers de l'ACEDLE, vol. 3 : 151-176.

Rinck, F. 2004. «Les difficultés d'étudiants dans la construction d'une problématique ». Pratiques, vol. 121/122:93-110.

Sockett, G. \& M. Kusyk. 2013. «L'apprentissage informel de l'anglais en ligne, nouvelle donne pour l'enseignement/apprentissage de l'anglais en Europe ». Recherche et pratiques pédagogiques en langues de spécialité, vol. $32 n^{\circ} 1: 75-$ 91.

Springer, C. 2009. «La dimension sociale dans le CECR : pistes pour scénariser, évaluer et valoriser l'apprentissage collaboratif ». Le français dans le monde recherches et applications, vol. $45: 25-34$.

Swales, J. M. 1990. Genre analysis: English in academic and research settings. Cambridge : Cambridge University Press.

Nathalie Gettliffe est maître de conférences au département de didactique des langues de 1'Université de Strasbourg. Ses recherches actuelles au sein de l'unité de recherche Linguistique Langue et parole (EA 1339) porte sur le français sur objectifs universitaires, l'utilisation des TICE en cours de langues et la formation réflexive des enseignants de langue.

$<$ ngettliffe@unistra.fr> 